\title{
Biological quality control for cardiopulmonary exercise testing in multicenter clinical trials
}

\author{
Janos Porszasz ${ }^{1 *}$, Susan Blonshine ${ }^{2}$, Robert Cao ${ }^{1}$, Heather A. Paden ${ }^{3}$, Richard Casaburi ${ }^{1}$ and Harry B. Rossiter ${ }^{1,4}$
}

\begin{abstract}
Background: Precision and accuracy assurance in cardiopulmonary exercise testing (CPET) facilitates multicenter clinical trials by maximizing statistical power and minimizing participant risk. Current guidelines recommend quality control that is largely based on precision at individual testing centers (minimizing test-retest variability). The aim of this study was to establish a multicenter biological quality control (BioQC) method that considers both precision and accuracy in CPET.

Methods: BioQC testing was 6-min treadmill walking at $20 \mathrm{~W}$ and $70 \mathrm{~W}$ (below the lactate threshold) with healthy non-smoking laboratory staff (15 centers; 16 months). Measurements were made twice within the initial 4 weeks and quarterly thereafter. Quality control was based on: 1) within-center precision (coefficient of variation [CV] for oxygen uptake $\left[\mathrm{V}_{2}\right]$, carbon dioxide output $\left[\dot{\mathrm{V}} \mathrm{CO}_{2}\right]$, and minute ventilation $[\dot{\mathrm{V}} \mathrm{E}]$ within $\pm 10 \%$ ); and 2) a criterion that $\dot{\mathrm{V}}_{2}$ at $20 \mathrm{~W}$ and $70 \mathrm{~W}$, and $\Delta \dot{\mathrm{V}} \mathrm{O}_{2} / \Delta \mathrm{WR}$ were each within $\pm 10 \%$ predicted. "Failed" BioQC tests (i.e., those outside the predetermined criterion) prompted troubleshooting and repeated measurements. An additional retrospective analysis, using a composite $z$-score combining both BioQC precision and accuracy of $\dot{\mathrm{V}}_{2}$ at $70 \mathrm{~W}$ and $\Delta \dot{\mathrm{VO}}_{2} / \Delta \mathrm{WR}$, was compared with the other methods.
\end{abstract}

Results: Of 129 tests ( 5 to 8 per center), 98 (76\%) were accepted by within-center precision alone. Within-center CV was $<9 \%$, but between-center $\mathrm{CV}$ remained high (9.6 to $12.5 \%$ ). Only 43 (33\%) tests had all $\mathrm{V}_{2}$ measurements within the $\pm 10 \%$ predicted criterion. However, a composite z-score of 0.67 identified 67 (52 \%) non-normal outlying tests, exclusion of which coincided with the minimum CV for CPET variables.

Conclusions: Study-wide BioQC using a composite z-score can increase study-wide precision and accuracy, and optimize the design and conduct of multicenter clinical trials involving CPET.

Trial registration: ClinicalTrials.gov identifier: NCT01072396; February 19, 2010.

Keywords: Calibration, Treadmill test, Pulmonary gas exchange, Z-score, Precision and accuracy

\section{Background}

Cardiopulmonary exercise testing (CPET) is a noninvasive, sensitive test to evaluate cardiopulmonary (patho-) physiology. CPET assesses the physiological basis of functional capacity and exercise intolerance, and plays a valuable role in diagnosis and clinical decision-making [1]. CPET is also used to test intervention efficacy, e.g.,

\footnotetext{
* Correspondence: porszasz@ucla.edu

${ }^{1}$ Rehabilitation Clinical Trials Center, Los Angeles Biomedical Research Institute at Harbor-UCLA Medical Center, 1124W Carson Street, Building CDCRC, Torrance, CA 90502, USA

Full list of author information is available at the end of the article
}

exercise training in cardiovascular disease [2, 3], pulmonary rehabilitation [4-8], and bronchodilator therapy in multicenter trials [9-11]. These applications require strong agreement between consecutively performed tests within and among investigative centers. However, precision (reproducibility, or test-retest variability) and accuracy (trueness) of CPET depend on interactions among: testing equipment variability, calibration, and maintenance; physiological factors; participants' cooperation, motivation, and effort during testing; and knowledge, skills, and training of laboratory personnel [12]. Most factors cannot be controlled by a simple system 
calibration, emphasizing the importance of standardization and quality control (QC). Clinicians can rely on trial results only if interpretation is not biased by measurement error $[12,13]$. Therefore, assurance of study-wide precision and accuracy has a major impact on the design and conduct of multicenter trials, by maximizing statistical discriminatory power, and minimizing laboratory burden and participant risk.

The American Thoracic Society (ATS)/American College of Chest Physicians (ACCP) [4], American Heart Association [3], and European Respiratory Society (ERS) $[7,12]$ have published CPET recommendations and standards. These give "best practice" for calibration and $\mathrm{QC}$, and provide typical coefficients of variation $(\mathrm{CV})$ for physiological measurements. ATS/ACCP [4] recommends a biological QC (BioQC) procedure [14] whereby a healthy subject on a stable diet performs regular exercise tests at work rates (WR) below the lactate threshold [12]. As physiologic responses are typically highly reproducible $[3,7,14-17]$, the use of healthy individuals performing BioQC can assure reproducibility of the integrated CPET measurement system for patient testing within and between testing centers [18]. Jones and Kane [19] used cycle ergometers and subjects who travelled to each participating center for testing, to demonstrate the efficacy of BioQC to minimize the study-wide CV for oxygen uptake $\left(\dot{\mathrm{VO}}_{2} ; 5.6 \%\right)$, carbon dioxide output $\left(\dot{\mathrm{V} C O}{ }_{2} ; 8.4 \%\right)$, and minute ventilation $(\dot{\mathrm{VE}} ; 8.2 \%)$ by excluding the least precise results. Similarly, while Gagnon et al. [20] showed good agreement among five testing centers for $\dot{\mathrm{V}} \mathrm{O}_{2, \text { peak }}$, this approach did not determine the precision and accuracy of measured exercise responses to a standardized protocol. Importantly, the intraclass correlation coefficient at low WR was $<0.7$ [20], emphasizing the need for QC of the relationship between mechanical and metabolic power output. Brawner et al. [21] used a standardized treadmill protocol, where quality assurance was accepted if at least two of three exercise stages fell within a "target range" for $\dot{\mathrm{V}}_{2}$. $\mathrm{Ra}$ ther than assuring the precision and accuracy of the individual mechanical-metabolic power coupling, the basis of the acceptability criteria (the "target range") in Brawner et al. [21] was wide, in part due to differences in weights of the volunteers used to develop the normative data.

In this study, we sought to establish new CPET BioQC acceptability criteria that considered both precision and accuracy of the mechanical-metabolic power coupling during a standardized treadmill protocol, and which would be suitable for quality assurance in multicenter studies.

We report the outcome of a precision-based approach to CPET QC in a multicenter trial (ClinicalTrials.gov identifier: NCT01072396) and propose new QC procedures based on both precision and accuracy to minimize variability in multicenter trials. The patient characterization and treatment phases of the parent trial have been reported $[22,23]$. Some of the results of this QC study have been previously reported in the form of an abstract [24].

\section{Methods}

Individuals involved in BioQC procedures

The primary independent Institutional Review Board (IRB), Chesapeake Research Review, Inc. (Columbia, MD, USA) approved the host trial protocol (NCT01072396) for five sites, which could utilize the study's central IRB, while the remaining 10 participating centers obtained individual IRB/ Independent Ethics Committee (IEC) approval (for details see Additional file 1: Table S1). The approved protocol included detailed exercise BioQC procedures in an Exercise and Quality Control Procedure Manual in accordance with ATS/ACCP recommendations [4]. During the trial, deidentified BioQC data from a non-smoking, healthy member of the laboratory staff at each center were submitted to a central reader. These laboratory staff members were required to fast and not consume caffeinated drinks for at least $2 \mathrm{~h}$ prior to testing, and their age, height, and weight (dressed, wearing shoes) were recorded. This manuscript describes an exempt retrospective study of this de-identified physiologic BioQC data from NCT01072396.

\section{Equipment used at the study centers}

Details of the CPET equipment, software versions, flow sensors, and treadmill details used in the study are shown in Additional file 2: Table S2. The treadmills were run from exercise software, with the exception of one center, where the treadmill was manually adjusted from its own controller using a preapproved procedure.

\section{Manual of procedures}

All centers selected to take part in the trial were provided with an Exercise and Quality Control Procedure Manual, which detailed a standardized approach to exercise testing, calibration, and QC. The main objectives of the manual were to: (1) provide information about the available guidelines to promote QC; (2) standardize technical procedures in CPET in order to minimize variation within and between participating centers; and (3) outline and standardize specific procedures involved in the clinical study.

\section{Staff training, and equipment calibration and verification}

Prior to enrolment of patients in the clinical trial, each participating center was visited by a consultant (TechEd Consultants, Inc., Mason, MI, USA) to evaluate and verify the equipment acceptability, and to standardize all QC and test procedures by providing specific training for staff. Training included an initial submaximal incremental exercise test 
and a BioQC constant work rate test. Details on staff training, and equipment calibration and verification are provided in Additional file 3.

A participating laboratory was only released for patient testing when all CPET technical and equipment performance qualifying criteria were met.

\section{BioQC procedures}

CPET systems were calibrated according to manufacturer's instructions immediately prior to BioQC testing (see Additional file 3). All centers measured $\dot{\mathrm{VO}}_{2}, \dot{\mathrm{V}} \mathrm{CO}_{2}$, and $\dot{V} E$ breath-by-breath via a mouthpiece, and heart rate from the electrocardiogram. The BioQC was a two-stage constant work rate treadmill exercise test. The 18-min protocol consisted of: $3 \mathrm{~min}$ of standing rest; $3 \mathrm{~min}$ of slow walking $(0.8 \mathrm{mph})$; and $6 \mathrm{~min}$ each at $20 \mathrm{~W}(1.0 \mathrm{mph})$ and $70 \mathrm{~W}$ (1.8 mph). Treadmill grades for each WR were calculated based on participant's clothed weight [25].

BioQC procedures were carried out at the on-site training visit, within 4 weeks post-training to verify validity of results, and quarterly thereafter for the duration of the study. Systems that required servicing (analyzer replacement, software updates, etc.) underwent an out-of-schedule BioQC test and patient testing was resumed only when the system passed the QC criteria. A maintenance and troubleshooting log was used to record all preventative maintenance activities, as well as faults and repairs (see Additional file 4).

\section{Accuracy and precision of gas exchange and ventilation measurements}

The BioQC tests were analyzed at a central reading site. Physiologic responses were submitted as 10 -s bin averages. Steady-state $\dot{\mathrm{V}} \mathrm{O}_{2}, \dot{\mathrm{V}} \mathrm{CO}_{2}$, and $\dot{\mathrm{V}} \mathrm{E}$ values were calculated by averaging the last $3 \mathrm{~min}$ of exercise at $20 \mathrm{~W}$ and $70 \mathrm{~W}$. Acceptability of each BioQC test was established by three methods: the central reader method, the z-score method, and the criterion method.

\section{Central reader method}

During the multicenter trial, BioQC acceptability was based upon within-center precision. A single central reader compared a BioQC result with the initiation and accumulated quarterly tests within each center. The BioQC was accepted if $\dot{\mathrm{VO}_{2}}, \dot{\mathrm{V}} \mathrm{CO}_{2}$, and $\dot{\mathrm{V} E}$ were within $\pm 10 \%$ of the initial value based on the expected normal variance [26-29] and ATS/ACCP requirements [4]. This method established precision over time (after an initial accuracy check at the staff-training visit) [4]. A measurement not meeting these criteria was repeated after troubleshooting (see Additional file 4).

\section{Z-score method}

After trial completion, all BioQC results were assessed en bloc to establish generalizable acceptability criteria.
Accuracy and precision of BioQC data were based on "position" and "slope" of the linear relationship between $\mathrm{VO}_{2}$ and WR [30], as predicted by the following [27]:

$$
\dot{\mathrm{V}} \mathrm{O}_{2}=(5.8 \times \text { weight }[\mathrm{kg}])+151+(10.1 \times \mathrm{W})
$$

Position was established from \% predicted $\dot{\mathrm{VO}}_{2}$ at $70 \mathrm{~W}$ $\left(\dot{\mathrm{V}} \mathrm{O}_{2,70 \mathrm{~W}}\right)$ and slope from \% predicted "functional gain" between $20 \mathrm{~W}$ and $70 \mathrm{~W}\left(\dot{\mathrm{VO}}_{2, \text { slope }}\right)$ based on a $\Delta \dot{\mathrm{VO}}_{2} / \Delta \mathrm{WR}$ of $10.1 \mathrm{~mL} / \mathrm{min} / \mathrm{W}[26,27]$, which has an established normal range of approximately $\pm 10 \%[28,29]$. Thus, accuracy was established by deviation from the predicted value, and precision from the standard deviation (SD) of all BioQC tests. A composite z-score in which position and slope were equally weighted was calculated:

$$
\begin{aligned}
\mathrm{z} & =\left(\mathrm{ABS}\left(\% \text { predicted } \dot{\mathrm{V}} \mathrm{O}_{2,70 \mathrm{w}}-100\right) / \mathrm{SD} \% \text { predicted } \dot{\mathrm{VO}}_{2,70 \mathrm{w}}\right) / 2 \\
& +\left(\mathrm{ABS}\left(\% \text { predicted } \dot{\mathrm{V}} \mathrm{O}_{2, \text { slope }}-100\right) / \mathrm{SD} \% \text { predicted } \dot{\mathrm{VO}}_{2, \text { slope }}\right) / 2
\end{aligned}
$$

where SD is the standard deviation of \% predicted for each variable. The Shapiro-Wilk test on the population of $\%$ predicted $\dot{\mathrm{V}} \mathrm{O}_{2,70 \mathrm{~W}}$ and $\dot{\mathrm{V}} \mathrm{O}_{2 \text {,slope }}$ measurements was used to identify z-score values associated with a systematic departure from normality (W statistic $P \leq 0.05$ ). As the composite $\mathrm{z}$-score criterion is based on $\%$ predicted values of all available tests across all centers, it was used to establish the minimum acceptability of both the precision and accuracy of CPET measurements.

\section{Criterion method}

Both acceptability methods (reader and z-score) were compared with the application of a rigid criterion that no single $\dot{\mathrm{V}} \mathrm{O}_{2}$ measurement $\left(\dot{\mathrm{VO}}_{2,20 \mathrm{w}}, \dot{\mathrm{V}} \mathrm{V}_{2,70 \mathrm{w}}\right.$, and $\left.\dot{\mathrm{V}} \mathrm{O}_{2 \text {,slope }}\right)$ should deviate by more than $\pm 10 \%$ from predicted. If any one measurement's deviation exceeded $\pm 10 \%$ predicted, the test was deemed to have failed QC.

\section{Statistical analyses}

Mean and SD were used to calculate CV for variables within and between centers. Departure from normality was assessed by the Shapiro-Wilk test. For multiple comparisons in one-way analysis of variance (ANOVA), the Student-Newman-Keuls test was used. Statistical significance was accepted if $P \leq 0.05$. All calculations and statistical analyses were performed using MS Excel (Redmond, WA, USA) and SigmaPlot 12 (Systat, San Jose, CA, USA).

\section{Results}

BioQC laboratory staff were males $(n=8$; age $41.4 \pm$ 11.4 years, weight $86.6 \pm 17.3 \mathrm{~kg}$, body mass index [BMI] $\left.28.0 \pm 4.3 \mathrm{~kg} / \mathrm{m}^{2}\right)$ and females $(n=7$; age $41.4 \pm 8.4$ years, 
weight $61.2 \pm 10.8 \mathrm{~kg}$, BMI $23.7 \pm 4.9 \mathrm{~kg} / \mathrm{m}^{2}$ ). The number of BioQC tests per center varied from five to eight, depending on the length of time the center was active in the trial $(16.3 \pm 3.1$ months [range 10 to 20 months]). This resulted in a total of 129 BioQC tests performed $(6.5 \pm 1.2$ per center), with each center having between 10 and 28 non-repeated pairs of BioQC tests. Overall, therefore, there were 523 unique paired differences of BioQC tests, which were used to establish the distribution properties of the measurements.

\section{Within-center variability - central reader method}

The central reader method accepted 98 (76\%) BioQC tests; 21 (24\%) initial tests required troubleshooting and repetition to meet QC acceptability criteria. At three centers, all tests were accepted. Four centers required one repeat test, two required two repeats, and five required three or more repeats to bring measurements within acceptable CV limits. Thus, across all centers, 31 repeated tests were necessary to bring BioQC measurements within acceptable CV limits (for a study-wide total of 129 tests). Further details on troubleshooting of the CPET systems during the study are provided in Additional file 4.

The within-center mean and variability of accepted BioQC tests are given in Table 1 . The most precise variables were $\dot{\mathrm{VO}}_{2}$ at $70 \mathrm{~W}(\mathrm{CV}=5.8 \%)$ and $\Delta \dot{\mathrm{VO}}_{2} / \Delta \mathrm{WR}$, which averaged $10.6 \pm 0.8 \mathrm{~mL} / \mathrm{min} / \mathrm{W}(\mathrm{CV}=5.8 \%)$. The least precise variable was $\dot{\mathrm{V} C O}{ }_{2}$ at $20 \mathrm{~W}(\mathrm{CV}=9.2 \%)$. The assumption that $\dot{\mathrm{VO}}_{2}$ at $70 \mathrm{~W}$ was below each individual's lactate threshold was supported by attainment of a steady-state within 6 min.

\section{Multicenter precision and accuracy Z-score method}

Study wide "position" accuracy of CPET measurements in the z-score method was assessed using $\dot{\mathrm{VO}}_{2,70 \mathrm{w}}$ because, of

Table 1 Within-center variability of gas exchange and ventilation measurement during treadmill exercise. Measurements were made at work rates of $20 \mathrm{~W}$ and $70 \mathrm{~W}$ in 98 reader-accepted biological quality control tests

\begin{tabular}{|c|c|c|c|}
\hline Variable & Work rate $(\mathrm{W})$ & Mean \pm SD & $\begin{array}{l}\text { Coefficient of } \\
\text { variation (\%) }\end{array}$ \\
\hline \multirow[t]{2}{*}{$\dot{\mathrm{V}} \mathrm{O}_{2}, \mathrm{~L} / \mathrm{min}$} & 20 & $0.73 \pm 0.15$ & 8.5 \\
\hline & 70 & $1.26 \pm 0.17$ & 5.8 \\
\hline \multirow[t]{2}{*}{$\dot{\mathrm{V}} \mathrm{CO}_{2}, \mathrm{~L} / \mathrm{min}$} & 20 & $0.59 \pm 0.12$ & 9.2 \\
\hline & 70 & $1.10 \pm 0.12$ & 7.2 \\
\hline \multirow[t]{2}{*}{$\dot{V} E, L / m i n$} & 20 & $18.5 \pm 4.2$ & 8.3 \\
\hline & 70 & $29.5 \pm 4.2$ & 6.3 \\
\hline$\Delta \dot{\mathrm{V}} \mathrm{O}_{2} / \Delta \mathrm{WR}, \mathrm{mL} / \mathrm{min} / \mathrm{W}$ & $\Delta 50$ & $10.6 \pm 0.8$ & 5.8 \\
\hline
\end{tabular}

$S D$ standard deviation, $\dot{V} O_{2}$ oxygen uptake, $\dot{V} \mathrm{CO}_{2}$ carbon dioxide output, $\dot{V} E$ minute ventilation, $\Delta \dot{V} \mathrm{O}_{2} / \Delta W R$ "functional gain" or the increase in $\dot{\mathrm{V}} \mathrm{O}_{2}$ per W the two steady-state $\dot{\mathrm{V}} \mathrm{O}_{2}$ measurements, $\dot{\mathrm{VO}}_{2,70 \mathrm{w}}$ showed less variability. Systematic inclusion of non-normal (outlying) BioQC measurements at $\dot{\mathrm{VO}}_{2,70 \mathrm{~W}}$ occurred above a z-score of 0.67 , while non-normal measurements of $\Delta \dot{\mathrm{VO}}_{2} / \Delta \mathrm{WR}$ occurred above a z-score of 0.75 (Fig. 1). Thus, using a critical z-score of 0.67, 62 (48 \%) tests were deemed acceptable. At $\mathrm{z}=0.67$, multicenter $\mathrm{CV}$ for $\dot{\mathrm{V}} \mathrm{O}_{2,20 \mathrm{~W}}, \dot{\mathrm{VO}}{ }_{2,70 \mathrm{~W}}$, and $\Delta \dot{\mathrm{VO}}_{2} / \Delta \mathrm{WR}$ were $6.2 \%, 4.7 \%$, and $6.0 \%$, respectively (Table 2 ). Accuracy was not different from the criterion method (see below), and close to $100 \%$ predicted in all cases (Table 2). The z-score was the only method for which final selected data for all $\mathrm{V}_{2}$ variables were normally distributed (Table 2). Composite $\mathrm{z}=$ 0.67 coincided with the lowest CV for absolute measurements in all three variables (Fig. 2a). Systematic increases in $\dot{\mathrm{VO}}_{2}$ measurement $\mathrm{CV}$ were observed at a z-score of $\sim 0.9$ (Fig. 2a) and CV exceeded ATS/ACCP guideline recommendations at a z-score of $\sim 1.0$ (Fig. 2b). The CV of \% predicted measurements increased with increasing $\mathrm{z}$ score, and $\mathrm{z}=0.67$ corresponded to a $\mathrm{CV}$ in all three variables less than $\sim 6 \%$ (Fig. 2 b) incorporating $\sim 50 \%$ of tests (Fig. 2c). Using the composite $\mathrm{z}$-score, a greater number of tests were excluded than using the central reader method (Table 2). Additionally, there was only $53 \%$ agreement in test acceptability between the central reader and composite z-score methods. Overall, precision and accuracy for $\dot{\mathrm{VO}}_{2}$ measurements using composite $\mathrm{z} \leq 0.67$ was greater than the central reader method and very similar to the criterion method, despite substantially more tests than the latter being deemed within acceptable limits.

\section{Criterion method}

When a criterion of $\pm 10 \%$ variability in all $\mathrm{VO}_{2}$ measurements (\% predicted $\dot{\mathrm{V}} \mathrm{O}_{2,20 \mathrm{~W}}, \dot{\mathrm{V}} \mathrm{O}_{2,70 \mathrm{~W}}$, and $\dot{\mathrm{V}}_{2, \text { slope }}$ ) was applied, only $43(33 \%)$ tests were acceptable. The criterion method resulted in greater precision (lower CV) and accuracy compared with the central reader method, but was not normally distributed in all variables (Table 2).

\section{Optimizing precision using distribution analysis of paired differences}

Analysis of 523 paired differences for $\dot{\mathrm{V}} \mathrm{O}_{2}, \dot{\mathrm{V}} \mathrm{CO}_{2}$, and $\dot{V} E$ is shown in Fig. 3. By including all tests, none of the variables were well described by a Gaussian distribution (Fig. 3, open circles). Whereas, for tests with composite $\mathrm{z} \leq 0.67$, all gas exchange and ventilation measurements (except $\dot{V} E$ at rest) were distributed normally (Fig. 3, closed circles). A composite z-score based on $\mathrm{VO}_{2}$ measurement alone was effective in also excluding tests with outlying, non-normal measurements in $\dot{\mathrm{V} C \mathrm{O}_{2}}$ and $\dot{\mathrm{V}}$. In all CPET variables, a QC method based on $\dot{\mathrm{VO}}_{2}$ precision and accuracy among all centers reduced data variability by $\sim 60 \%$ compared with using no QC method, and by $\sim 50 \%$ compared with the central reader method. 


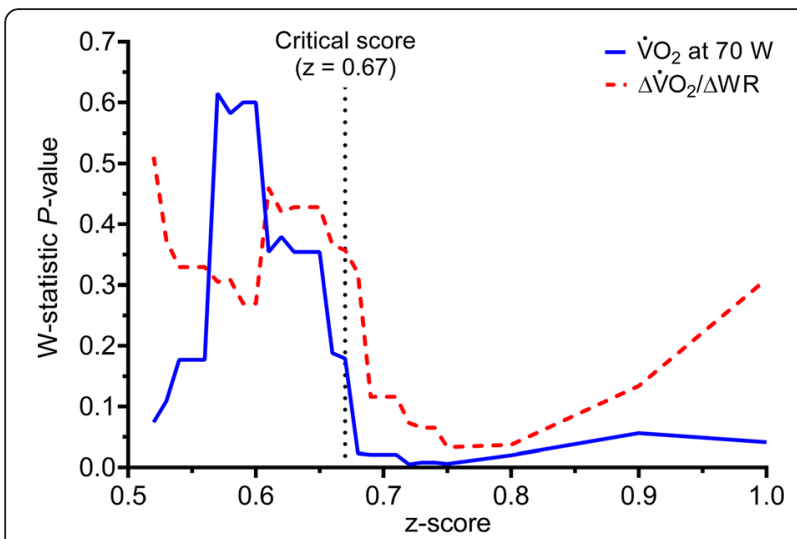

Fig. 1 Effect of z-score on normality of oxygen uptake $\left(\mathrm{V}_{2}\right)$ measurement distribution. Normalcy of biological quality control test measurements selected on the basis of a composite $z$-score. Above a critical z-score of 0.67 , the W statistic drops dramatically and the distribution of paired differences becomes non-normal for $\dot{\mathrm{V}}_{2}$ at $70 \mathrm{~W}$

\section{Discussion}

To our knowledge, this is the first study to establish study-wide precision and accuracy for CPET measurements in multicenter trials, based on normalcy of the coupling between mechanical and metabolic power output. We found: 1) the CV of CPET measurements was reduced using the central reader assessment of BioQC exercise tests to minimize within-center variability; 2) a rigid application of a $\pm 10 \%$ predicted $\dot{\mathrm{VO}}_{2}$ cut-off criterion excluded $67 \%$ of all measurements, some of which were within the normal distribution of accurate tests; and 3) the application of a composite z-score to identify measurements lying outside normal limits increased the precision and accuracy of multicenter trial CPET measurements by $\sim 50 \%$.

\section{Efficacy of CPET QC based on within-center precision}

Acceptance rate by the central reader method for all BioQC tests was $76 \%$. The BioQC process identified measurement errors in 12 of 15 centers before measurements of study patients were made in the parent clinical trial. In six centers, measurement error was resolved by standard troubleshooting approaches, and required only one or two additional BioQC tests to demonstrate resolution. The central reader method excluded outlying tests based on reproducibility, and effectively excluded tests departing from normality. Analysis of all tests showed non-normal distribution, whereas including only tests accepted by the central reader assessment resulted in a data set that was not significantly different from a normal distribution (Table 2). The central reading process reduced within-center $\mathrm{CV}$ for gas exchange and ventilation measurements to within the range 5.8 to

Table 2 Characteristics of variability of oxygen uptake $\left(\mathrm{V}_{2}\right)$ measurements during 129 biological quality control tests, using three different quality control methods

\begin{tabular}{|c|c|c|c|c|c|c|}
\hline Variable & Work rate $(\mathrm{W})$ & $\begin{array}{l}\text { Number } \\
\text { (\% of all tests) }\end{array}$ & $\begin{array}{l}\text { Mean } \pm \text { SD } \\
\text { (\% predicted) }\end{array}$ & $\begin{array}{l}\text { Median } \\
\text { (\% predicted) }\end{array}$ & Coefficient of variation (\%) & Normality (Shapiro-Wilk) \\
\hline \multicolumn{7}{|l|}{$\overline{\mathrm{V}} \mathrm{O}_{2}$ at $20 \mathrm{~W}$} \\
\hline All tests & 20 & $129(100)$ & $92.1 \pm 12.2$ & 93.5 & 14.3 & Passed \\
\hline Criterion $^{a}$ & 20 & $43(33)$ & $96.1 \pm 5.4$ & 94.8 & 5.6 & $P<0.001$ \\
\hline Reader $^{b}$ & 20 & $98(76)$ & $91.9 \pm 11.5^{d}$ & 93.1 & 12.5 & Passed \\
\hline Composite z-score & 20 & $62(48)$ & $95.8 \pm 5.9$ & 95.0 & 6.2 & Passed \\
\hline \multicolumn{7}{|l|}{$\dot{\mathrm{V}} \mathrm{O}_{2}$ at $70 \mathrm{~W}$} \\
\hline All tests & 70 & $129(100)$ & $97.5 \pm 11.0$ & 97.7 & 11.3 & Passed \\
\hline Criterion $^{a}$ & 70 & $43(33)$ & $98.4 \pm 4.1$ & 98.1 & 4.2 & Passed \\
\hline Reader $^{\mathrm{b}}$ & 70 & $98(76)$ & $97.6 \pm 9.4$ & 97.1 & 9.6 & Passed \\
\hline Composite $z$-score ${ }^{c}$ & 70 & $62(48)$ & $99.0 \pm 4.6$ & 98.4 & 4.7 & Passed \\
\hline \multicolumn{7}{|l|}{$\Delta \dot{\mathrm{VO}} \mathrm{O}_{2} / \Delta \mathrm{WR}$} \\
\hline All tests & $\Delta 50$ & $129(100)$ & $105.7 \pm 13.6$ & 105.8 & 12.9 & $P=0.006$ \\
\hline Criterion $^{a}$ & $\Delta 50$ & $43(33)$ & $102.0 \pm 5.4$ & 102.5 & 5.3 & Passed \\
\hline Reader $^{\mathrm{b}}$ & $\Delta 50$ & $98(76)$ & $106.3 \pm 11.4$ & 105.1 & 10.7 & $P<0.001$ \\
\hline Composite $z$-score ${ }^{c}$ & $\Delta 50$ & $62(48)$ & $103.9 \pm 6.2$ & 104.3 & 6.0 & Passed \\
\hline
\end{tabular}

$S D$ standard deviation, $\dot{V} O_{2}$ oxygen uptake, $\dot{V} \mathrm{CO}_{2}$ carbon dioxide output, $\dot{V} E$ minute ventilation, $\Delta \dot{V} O_{2} / \Delta W R$ "functional gain" or the increase in $\dot{V} \mathrm{O}_{2}$ per W

${ }^{a}$ Criterion method was based on $\dot{\mathrm{V}}_{2,20 \mathrm{w}}, \dot{\mathrm{V}} \mathrm{O}_{2,70 \mathrm{w}}$ and $\dot{\mathrm{V}}_{2, \text { slope }}$ being within $\pm 10 \%$ predicted

${ }^{\mathrm{b}}$ Reader method was based on $\dot{\mathrm{VO}}_{2}, \dot{\mathrm{V}} \mathrm{CO}_{2}$, and $\dot{\mathrm{V} E}$ within $\pm 10 \%$ of the initial value

cComposite $z$-score of 0.67 , based on deviation of $\dot{\mathrm{V}}_{2,70 \mathrm{w}}$ and $\dot{\mathrm{VO}}_{2, \text { slope }}$ from predicted, with knowledge of SD from all BioQC tests

${ }^{\mathrm{d}} P<0.05$ Student-Newman-Keuls multiple comparison test within the $\dot{\mathrm{V}}_{2}$ at $20 \mathrm{~W}$ 

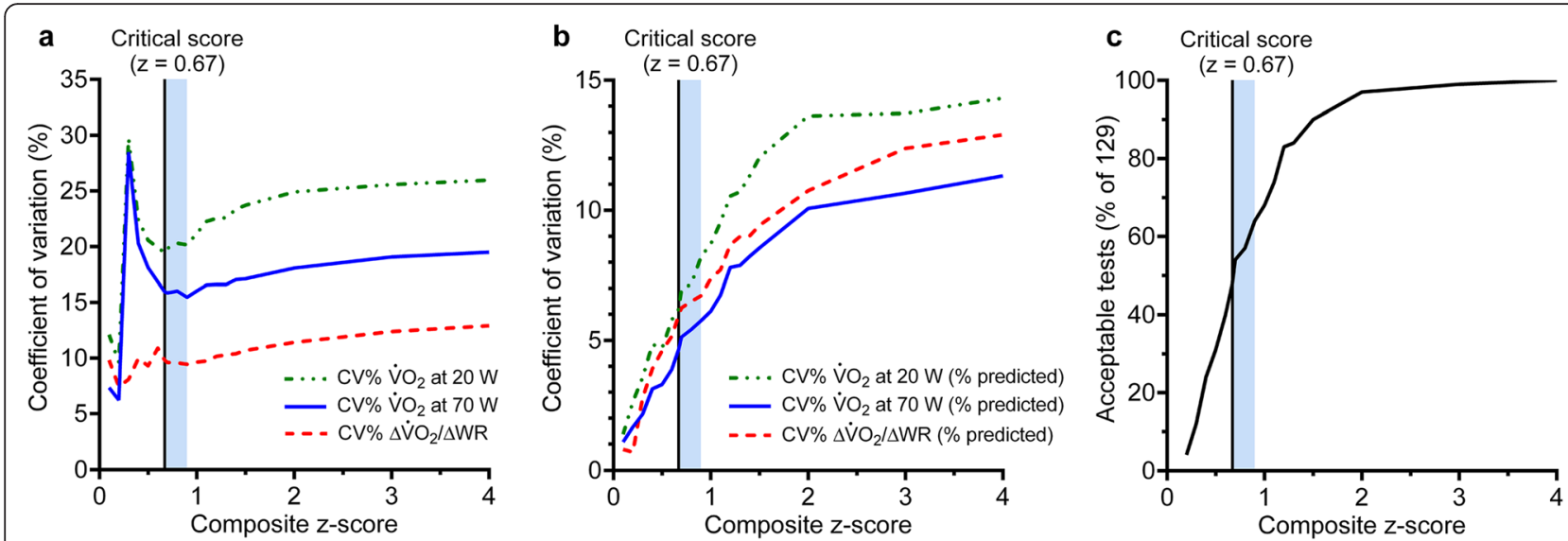

Fig. 2 Effect of $z=0.67$ cut-off on the $C V$ and number of accepted CPETs. a Coefficient of variation (CV) of the absolute oxygen uptake $\left(\mathrm{V}_{2}\right)$ at $20 \mathrm{~W}$, $70 \mathrm{~W}$, and the increase in $\dot{\mathrm{V}} \mathrm{O}_{2}$ per $\mathrm{W}\left(\Delta \dot{\mathrm{V}} \mathrm{O}_{2} / \Delta \mathrm{WR}\right)$, at different z-scores. b $\mathrm{CV}$ of \% predicted $\dot{\mathrm{V}} \mathrm{O}_{2}$ at $20 \mathrm{~W}, 70 \mathrm{~W}$, and $\Delta \dot{\mathrm{V}} \mathrm{O}_{2} / \Delta \mathrm{WR}$, at different z-scores. c $\%$ of acceptable tests $(n=129)$, at different $z$-scores. The shaded area is the approximate range of $z$-scores $(0.67$ to 0.9$)$ over which absolute measurement CV was minimized (based on panel $\mathbf{a}$, and transposed into panels $\mathbf{b}$ and $\mathbf{c}$ ). The critical z-score is the minimum value for which all measurements are normally distributed. It is noted that the absolute CV (panel a) depends on both variability in measurements and differences in weight of individuals performing the biological quality control tests (absolute $\mathrm{VO}_{2}$ at $20 \mathrm{~W}$ and $70 \mathrm{~W}$ treadmill walking is dependent on weight). Weight of individuals did not significantly vary during the trial. Despite this, the absolute $\mathrm{CV}$ is useful to isolate the z-score range at which the minimum CV occurred (shaded bar). The variability due to measurement differences among centers is better assessed using \% predicted values (panel b)

$9.2 \%$ (Table 1 ), which is within the range generally accepted for CPET studies [3, 4, 12, 31, 32].

Application of a central BioQC reader, therefore, provided a beneficial addition that reduced measurement variability in the multicenter trial. However, while within-center variability was below the upper limits recommended by ATS/ACCP [4] ( 10\%), there remained variability between different centers. The between-center $\mathrm{CV}$ for gas exchange measurements of all centrally accepted BioQC tests ranged from 9.6 to $12.5 \%$ (Table 2). This residual between-center variability effectively lessened the benefit of the BioQC method, and weakened the statistical power to demonstrate a given change in CPET outcome measures in the parent clinical trial [23].

\section{CPET QC based on study-wide precision and accuracy}

To our knowledge, only one previous study attempted a QC regimen based on both precision and accuracy [21]. However, the accuracy criterion used was wide $( \pm 25 \%)$ because the approach did not account for differences in mechanical power output during treadmill walking at given speed/grade combinations among individuals differing in weight. Therefore, to develop an approach to CPET QC in multicenter trials that included study-wide precision and accuracy in the coupling of mechanical to metabolic power, we applied two different BioQC methods. The first, a simple criterion approach, rigidly excluded BioQC tests in which accuracy of any one $\mathrm{VO}_{2}$ measurement $\left(\dot{\mathrm{VO}}_{2,20 \mathrm{~W}}, \dot{\mathrm{V}} \mathrm{O}_{2,70 \mathrm{w}}\right.$, and $\left.\dot{\mathrm{V}} \mathrm{O}_{2 \text {,slope }}\right)$ lay outside 90 to $110 \%$ of the predicted value at the calculated power output. This approach appears inherently sensible, in that measurements outside this $\pm 10 \%$ range (roughly based on guideline recommendations $[4,31,32])$ are considered as outlying, and thus excluded. However, a limitation is that a relatively small error in only one variable causes a failing BioQC test, even if all other measurements are within the tolerable limit. The very low acceptability rate of tests across the 15 centers (33\%) makes application of this criterion impractical. Indeed, seven study centers would have been completely excluded from the trial, despite reporting demonstrably accurate measurements based on the retrospective analysis of the normal distribution of the BioQC measurements (Fig. 3). Thus, while the rigid criterion dramatically improved the CV of CPET measurements, it excluded data that were within the normal distribution of measurement variability (e.g., Fig. 1, Table 2).

Therefore, we developed a method that allowed small variability outside the 90 to $110 \%$ range for predicted $\dot{\mathrm{V}} \mathrm{O}_{2}$, but could successfully identify outlying, non-normal measurements and reduce between-center measurement $\mathrm{CV}$. The composite z-score considered equally, the relative deviation from the mean of "position" ( $\left.\mathrm{VO}_{2,70 \mathrm{w}}\right)$ and "slope" $\left(\Delta \dot{V}_{2} / \Delta W R\right)$ of the highly predictable relationship between $\dot{\mathrm{VO}}_{2}$ and WR. By combining error distribution of these two variables, a small deviation from predicted (i.e., between $\sim 87 \%$ and $113 \%$ ) in one measurement was allowed as long as the other was accurate. We found this method able to strongly predict systematic deviations in non-normal measurements above a composite z-score of 0.67 (based on SD of all tests; Fig. 1). In addition, composite $\mathrm{z}$-score of 0.67 coincided with local minima in CV of absolute $\dot{\mathrm{VO}}_{2}$ measurements (Fig. 2a) and resulted in a study-wide CV of $\sim 6 \%$ (Fig. 2b). 


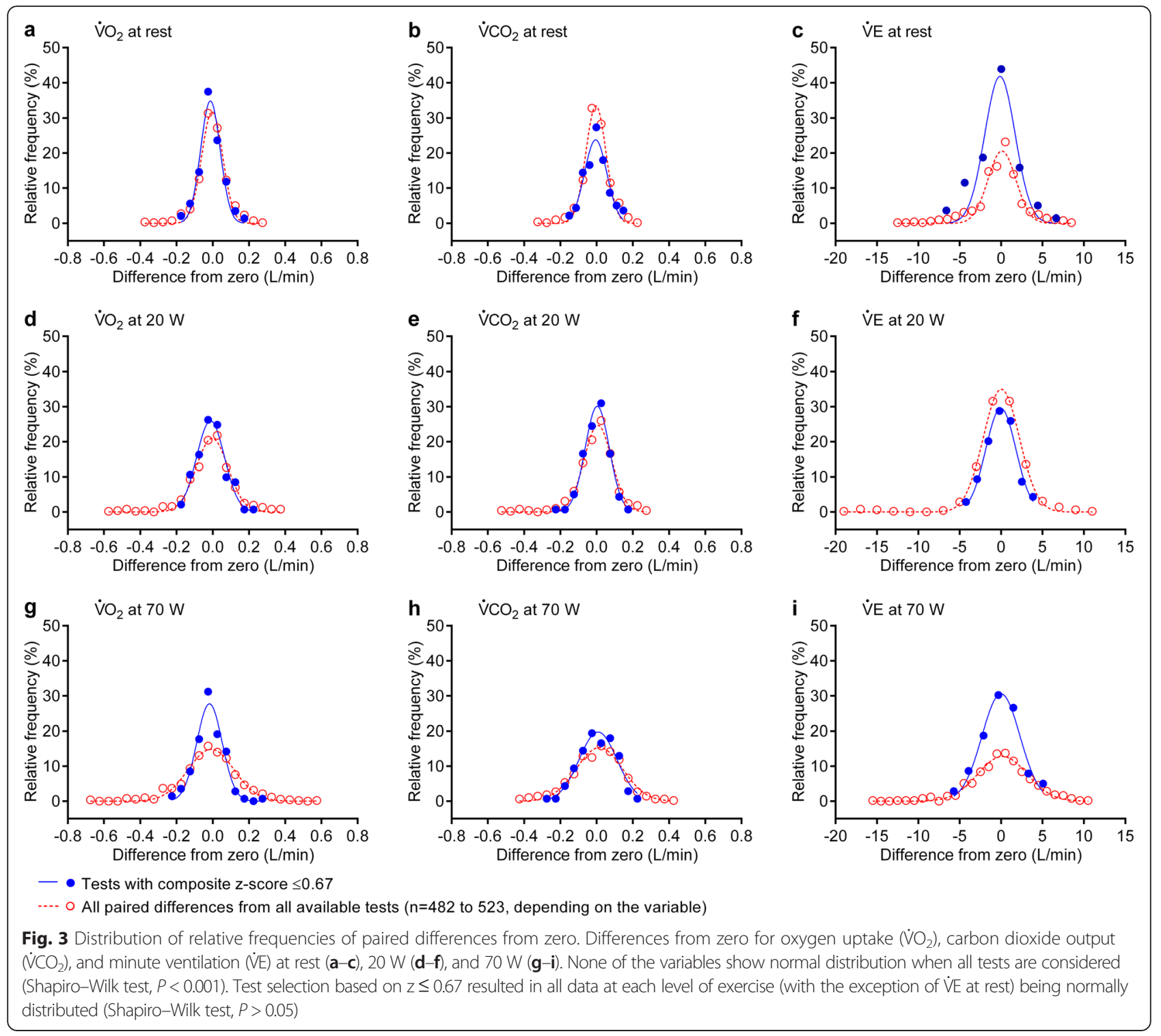

Using $\mathrm{z}=0.67$ we were able to identify that $52 \%$ of the BioQC tests lay outside the normal distribution of $\dot{\mathrm{V}}_{2}$ measurements. While this approach excluded more tests than the central reading method, this combined precision- and accuracy-based method achieved three main benefits. Firstly, it had a strong agreement with the rigid criterion-based approach (84\% agreement between methods). Secondly, it had a relatively high acceptance rate (48\%) without compromising narrow measurement $\mathrm{CV}$ compared with the criterion method (Table 2). Lastly, it had a low CV of CPET measurements; 50\% lower $\mathrm{CV}$ than that compared with central precisionbased QC approaches that form the basis of guideline recommendations. This latter point is of considerable importance for the design and conduct of multicenter clinical trials with CPET measurement outcomes. By applying a z-score-based BioQC method across all centers, we suggest that measurement variability can be reduced by $\sim 50 \%$, providing an increase in statistical power to detect changes in CPET measurements. Regular BioQC tests are not onerous and, until a larger BioQC data population is established, any CPET laboratory seeking to implement a $\mathrm{QC}$ procedure may simply apply the zscore criterion using equation 2, and the study-wide population SD values established in this study $(11.0 \%$

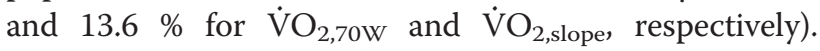
While we found the optimal z-score at 0.67 , based on distribution normality, the $\mathrm{CV}$ of absolute $\mathrm{VO}_{2}$ measurements remained close to the minimum up to a $\mathrm{z}$-score of $\sim 0.90$, corresponding to $\sim 65 \%$ of all tests and a CV for $\%$ predicted below ATS/ACCP guidelines (which occurred at z-score $\sim 1.0$ ) [4]. Further research is required to determine more precisely the optimal $\mathrm{z}$-score within the range of $\sim 0.67$ to $\sim 0.90$ that balances requirements 
of normality, minimized CV and the number of accepted tests to inform CPET studies. Nevertheless, while using a combined z-score of 0.67 would minimize CPET measurement variability, any power calculations for future clinical trials should also account for the response variability inherent in the clinical population studied. Thus, the combined z-score approach maximizes statistical discriminatory power within multicenter trials and minimizes laboratory testing burden and study participant risk.

\section{Strategies to minimize measurement variability}

We found a relatively high rate of measurement error over 16 months. Importantly, 8 of 15 centers (53\%) required at least one additional validation procedure after an initial failing BioQC test. Each failure triggered a CPET-system troubleshooting process, which included site technicians and central support to identify the error source. Most required involvement of the system manufacturer and eventually led to major equipment service, emphasizing the need for regular maintenance.

In addition, this justifies the recommendation for frequent and rigorously evaluated QC methods in order to prevent large unexplained variability in CPET measurements. The BioQC process used here also identified equipment error prior to equipment failure, and allowed centers to address failing components of CPET systems before trial-related measurements were scheduled. Overall, our results support the view that systematic BioQC is needed to achieve satisfactorily accurate and precise data in multicenter trials employing CPET.

\section{Limitations}

A potential limitation relates to the accurate estimation of treadmill WR. External WR is calculated considering a subject's weight [33]; but does not account for the inertia associated with body movements while walking [25]. These inertial components increase with weight, which may reduce the accuracy of calculated WR and predicted $\dot{\mathrm{VO}}_{2}$ value upon which BioQC is based. A similar phenomenon occurs in cycle ergometry, where $\dot{\mathrm{VO}}_{2}$ is influenced by pedaling frequency. However, a change in treadmill speed between $20 \mathrm{~W}$ and $70 \mathrm{~W}(1.0$ $\mathrm{mph}$ to $1.8 \mathrm{mph}$ ) requires an obligatory cadence increase and thus variable internal work; in cycling, cadence can be effectively controlled [34, 35]. One solution would be to recruit BioQC subjects who are similar in weight to potential trial patients. Another solution would be to use calculations for treadmill WR that incorporate kinetic energy $\left(\mathrm{mv}^{2}\right)$ instead of momentum (mv).

Another limitation is that we used an equation to predict metabolic rate that was originally developed for cycle ergometry. However, within the speed range used in this study, measured and predicted metabolic rates show strong agreement [25]. $\dot{\mathrm{V}} \mathrm{O}_{2}$ prediction was based on a $\Delta \dot{\mathrm{VO}} \mathrm{O}_{2} / \Delta \mathrm{WR}$ of $10.1 \mathrm{~mL} / \mathrm{min} / \mathrm{W}[26,27]$. A range of studies support this value, e.g., $10.2 \pm 1.0 \mathrm{~mL} / \mathrm{min} / \mathrm{W}$ [28] and $9.9 \pm 0.7 \mathrm{~mL} / \mathrm{min} / \mathrm{W}$ [29], although it is recognized that a greater value may be seen in endurancetrained individuals [26]. While, in this study we found that $\Delta \dot{\mathrm{VO}}_{2} / \Delta \mathrm{WR}$ averaged $10.6 \mathrm{~mL} / \mathrm{min} / \mathrm{W}$, this mean was derived from only 15 individuals who performed the BioQC and was within the normal range. We found that post hoc adjustment of the target $\Delta \dot{\mathrm{VO}}_{2} / \Delta \mathrm{WR}$ between $10.1 \mathrm{~mL} / \mathrm{min} / \mathrm{W}$ and $10.6 \mathrm{~mL} / \mathrm{min} / \mathrm{W}$ excluded only one additional BioQC test and had no effect on the optimal z-score range. Nevertheless, equations to better calculate treadmill WR to improve accuracy of the $\Delta \dot{\mathrm{V} O} \mathrm{O}_{2} / \Delta \mathrm{WR}$ target, or using exercise modalities such as cycling where WR can be better controlled, would likely further improve precision and accuracy provided by the composite z-score BioQC method.

The BioQC method relies on the attainment of steadystate metabolic responses below the lactate threshold at $70 \mathrm{~W}$. This may require verification by an additional incremental exercise test for non-invasive lactate threshold estimation, and/or the use of a lower WR for less aerobically fit or smaller individuals.

The QC method assesses instrumental measurement precision and accuracy (as opposed to physiologic variability) from submaximal steady-state CPET measurements, because the variability of predicted values for healthy participants is low within this domain. However, clinical trials typically assess both submaximal and maximal values from CPET measurements. Therefore, the instrumental measurement precision determined in this study may not necessarily reflect the instrumental precision of peak measurements, where breathing frequency is greater, and the response times of the gas analyzers become increasingly important. Nevertheless, quality assurance of the integrated CPET measurement system linking the mechanical and metabolic power output within the submaximal domain should also contribute to improving assurance of multicenter instrumental precision and accuracy of maximal CPET measurements.

\section{Conclusions}

A central precision-based QC procedure for multicenter studies with CPET as an outcome, reduced measurement variability within center, but was not sufficient to assure between-center measurement accuracy. Thus, we established the distribution of measurements linking mechanical and metabolic power output during multicenter CPET testing, and used this to develop a composite z-score-based method to assess accuracy and precision of CPET measurements. Based on 129 moderate intensity BioQC 
exercise tests in healthy laboratory staff across 15 centers and 16 months, we found that a composite z-score of 0.67 was able to detect non-normal (outlying) CPET measurements and trigger CPET system troubleshooting, enabling a reduction of multicenter measurement variability by $\sim 50 \%$. The measurement distribution and zscore method established in this study may be applied to future multicenter studies where CPET variables are measured. Thus, a study-wide precision- and accuracybased QC process is required to optimize the design, sample size, and conduct of multicenter clinical trials involving CPET measurements.

\section{Availability of data and materials Not applicable.}

\section{Additional files}

Additional file 1: Table S1. Independent Ethics Committees and Institutional Review Boards for ClinicalTrial.gov: NCT01072396. (PDF 71 kb)

Additional file 2: Table S2. Equipment used at the study centers. (PDF $18 \mathrm{~kb}$ )

Additional file 3: Staff training, and equipment calibration and verification. (PDF $75 \mathrm{~kb}$ )

Additional file 4: Maintenance and troubleshooting log, and troubleshooting of CPET systems consequent to a failing BioQC test. (PDF $64 \mathrm{~kb}$ )

\section{Abbreviations}

ATS: American Thoracic Society; ACCP: American College of Chest Physicians: BioQC: Biological quality control; BMI: Body mass index; $\dot{\mathrm{V}} \mathrm{CO}_{2}$ : Carbon dioxide output; CPET: Cardiopulmonary exercise testing; CV: Coefficient of variation; ERS: European Respiratory Society; IRB: Institutional Review Board; $\mathrm{mv}^{2}$ : Kinetic energy; VE: Minute ventilation; mv: Momentum; $\mathrm{VO}_{2}$ : Oxygen uptake; QC: Quality control; SD: Standard deviation; WR: Work rate.

\section{Competing interests}

Janos Porszasz reports grants, personal fees, and non-financial support from Boehringer Ingelheim, Inc., grants from Actelion, and personal fees from Actelion and Allergan during the conduct of the study. In addition, Dr. Porszasz has a patent US 7,628,732 issued, and a patent US 7,927,251 issued.

Susan Blonshine reports personal fees from Boehringer Ingelheim during the conduct of the study and personal fees from Boehringer Ingelheim outside the submitted work

Robert Cao was a consultant for Boehringer Ingelheim Pharmaceuticals, Inc Heather A Paden is an employee of Boehringer Ingelheim Pharmaceuticals, Inc. Richard Casaburi reports grants from Los Angeles Biomedical Research Institute and personal fees from Boehringer Ingelheim during the conduct of this study. He reports grants from Boehringer Ingelheim, Forest, GlaxoSmithKline, and Novartis, and personal fees from AstraZeneca, Boehringer Ingelheim, Forest, GlaxoSmithKline, Novartis, and Pfizer outside the submitted work. Harry B Rossiter declares that he has no competing interests.

\section{Authors' contributions}

JP, SB, HP, RCas, and HBR participated in the conception, hypotheses delineation, and design of the study. JP, SB, and RCao collected the study data. JP, RCao, and HBR performed the statistical analyses. JP, HBR, and RCas interpreted the results. JP, SB, RCas, and HBR drafted the manuscript. All authors reviewed manuscript drafts, and read and approved the final version

\section{Acknowledgments}

The authors wish to express their indebtedness to all laboratory staff who sacrificed their time and effort to comply with the special requirements, and spent significant time on performing these tests without complaint in order to achieve the highest possible quality in this trial. Furthermore, we would also like to acknowledge all center principal investigators and personnel who contributed their time and effort to this study. This work was supported by Boehringer Ingelheim Pharmaceuticals, Inc. (BIPI) and Pfizer Inc. Writing, editorial support, and formatting assistance was provided by Jane M. Gilbert, BSc, CMPP, of Envision Scientific Solutions, which was contracted, and compensated by BIPI and Pfizer Inc for these services.

\section{Author details}

${ }^{1}$ Rehabilitation Clinical Trials Center, Los Angeles Biomedical Research Institute at Harbor-UCLA Medical Center, 1124W Carson Street, Building CDCRC, Torrance, CA 90502, USA. ${ }^{2}$ TechEd Consultants, Inc., Mason, MI, USA. ${ }^{3}$ Boehringer Ingelheim Pharmaceuticals Inc., Ridgefield, CT, USA. ${ }^{4}$ Faculty of Biological Sciences, University of Leeds, Leeds, UK.

Received: 2 September 2015 Revised: 6 January 2016

Accepted: 11 January 2016

Published online: 16 January 2016

\section{References}

1. Wasserman K, Hansen JE, Sue DY, Stringer WW, Whipp BJ. Clinical applications of cardiopulmonary exercise testing. In: Principles of Exercise Testing and Interpretation Including Pathophysiology and Clinical Applications. 4th ed. Philadelphia: Lippincott Williams \& Wilkins; 2005. p. 198-241.

2. Mezzani A, Corrà U, Giordano A, Colombo S, Psaroudaki M, Giannuzzi P. Upper intensity limit for prolonged aerobic exercise in chronic heart failure. Med Sci Sports Exerc. 2010;42(4):633-9.

3. Myers J, Arena R, Franklin B, Pina I, Kraus WE, McInnis K, et al. Recommendations for clinical exercise laboratories: a scientific statement from the American Heart Association. Circulation. 2009:119(24):3144-61.

4. American Thoracic Society (ATS)/American College of Chest Physicians (ACCP). ATS/ACCP Statement on cardiopulmonary exercise testing. Am J Respir Crit Care Med. 2003;167(2):211-77.

5. Casaburi R, Porszasz J, Burns MR, Carithers ER, Chang RS, Cooper CB. Physiologic benefits of exercise training in rehabilitation of patients with severe chronic obstructive pulmonary disease. Am J Respir Crit Care Med. 1997;155(5):1541-51.

6. Emtner M, Porszasz J, Burns M, Somfay A, Casaburi R. Benefits of supplemental oxygen in exercise training in nonhypoxemic chronic obstructive pulmonary disease patients. Am J Respir Crit Care Med. 2003;168(9):1034-42.

7. Palange P, Ward SA, Carlsen KH, Casaburi R, Gallagher CG, Gosselink R, et al. Recommendations on the use of exercise testing in clinical practice. Eur Respir J. 2007:29(1):185-209.

8. Vogiatzis I. Strategies of muscle training in very severe COPD patients. Eur Respir J. 2011;38(4):971-5.

9. Maltais F, Celli B, Casaburi R, Porszasz J, Jarreta D, Seoane B, et al. Aclidinium bromide improves exercise endurance and lung hyperinflation in patients with moderate to severe COPD. Respir Med. 2011;105(4):580-7.

10. Neder JA, Fuld JP, Overend T, Thirlwell J, Carter R, Stevenson R, et al. Effects of formoterol on exercise tolerance in severely disabled patients with COPD. Respir Med. 2007;101(10):2056-64.

11. O'Donnell DE, Flüge T, Gerken F, Hamilton A, Webb K, Aguilaniu B, et al. Effects of tiotropium on lung hyperinflation, dyspnoea and exercise tolerance in COPD. Eur Respir J. 2004:23(6):832-40

12. Porszasz J, Stringer W, Casaburi R. Equipment, measurements and quality control in clinical exercise testing. In: Ward S, Palange P, editors. European Respiratory Monograph, No 40. Volume 12. Sheffield, UK: European Respiratory Society; 2007. p. 108-28.

13. Lamarra N, Whipp B. Measurement of pulmonary gas exchange. In: Maud PJ, Foster C, editors. Physiological Assessment of Human Fitness. Champaign: Human Kinetics; 1995. p. 19-35.

14. Revill SM, Morgan MD. Biological quality control for exercise testing. Thorax 2000;55(1):63-6.

15. Arena $R$, Peberdy MA. Short-term reliability of oxygen uptake on-kinetics in apparently healthy subjects. J Cardiopulm Rehabil. 2006:26(4):219-23.

16. Hansen JE, Sun XG, Yasunobu Y, Garafano RP, Gates G, Barst RJ, et al. Reproducibility of cardiopulmonary exercise measurements in patients with pulmonary arterial hypertension. Chest. 2004;126(3):816-24.

17. Johnston KN, Jenkins SC, Stick SM. Repeatability of peak oxygen uptake in children who are healthy. Pediatr Phys Ther. 2005;17(1):11-7. 
18. Porszasz J, Woodhouse LI, Casaburi R, Nguyen AHC, Tsurugaya H, Whipp BJ. Use of physiological calibration to validate gas exchange measurements during exercise. Med Sci Sports Exerc. 2004;36(5):S27.

19. Jones NL, Kane JW. Quality control of exercise test measurements. Med Sci Sports. 1979;11(4):368-72.

20. Gagnon J, Province MA, Bouchard C, Leon AS, Skinner JS, Wilmore JH, et al. The HERITAGE Family Study: quality assurance and quality control. Ann Epidemiol. 1996;6(6):520-9.

21. Brawner CA, Ehrman JK, Aldred H, Schairer JR, Keteyian SJ. Quality assurance and cardiopulmonary exercise testing in clinical trials. J Card Fail. 2008:14(4):283-9.

22. O'Donnell DE, Maltais F, Porszasz J, Webb KA, Albers FC, Deng Q, et al. The continuum of physiological impairment during treadmill walking in patients with mild-to-moderate COPD: patient characterization phase of a randomized clinical trial. PloS one. 2014:9(5):e96574

23. Casaburi R, Maltais F, Porszasz J, Albers F, Deng Q, lqbal A, et al. Effects of tiotropium on hyperinflation and treadmill exercise tolerance in mild to moderate chronic obstructive pulmonary disease. Ann Am Thorac Soc 2014;11(9):1351-61.

24. Blonshine B, Cao R, Casaburi R, Paden H, Porszasz J. Results from a multi-center cardiopulmonary exercise physiological quality control protocol. American Thoracic Society 2012 International Conference, May 18-23, 2012, San Francisco, CA, USA. Am J Resp Crit Care Med, Vol. 185, Meeting Abstracts, 2012. A43 COPD: Functional Assessment and Exercise. p. A1545.

25. Porszasz J, Casaburi R, Somfay A, Woodhouse LJ, Whipp BJ. A treadmill ramp protocol using simultaneous changes in speed and grade. Med Sci Sports Exerc. 2003;35(9):1596-603.

26. Wasserman K, Hansen J, Sue D, Stringer W, Whipp B. Oxygen cost of work. In: Principles of Exercise Testing and Interpretation Including Pathophysiology and Clinical Applications. 4th ed. Philadelphia: Lippincott Williams and Wilkins; 2005. p. 19.

27. Wasserman K, Whipp BJ. Exercise physiology in health and disease. Am Rev Respir Dis. 1975;112(2):219-49.

28. Hansen JE, Sue DY, Wasserman K. Predicted values for clinical exercise testing. Am Rev Respir Dis. 1984:129(2 Pt 2):S49-55.

29. Wasserman K, Sue D. Coupling of external to cellular respiration. In: Wasserman K, editor. Exercise Gas Exchange in Heart Disease. New York: Futura Publishing; 1996.

30. Hansen JE, Casaburi R, Cooper DM, Wasserman K. Oxygen uptake as related to work rate increment during cycle ergometer exercise. Eur J Appl Physiol Occup Physiol. 1988;57(2):140-5.

31. Gibbons RJ, Balady GJ, Bricker JT, Chaitman BR, Fletcher GF, Froelicher VF, et al. ACC/AHA 2002 guideline update for exercise testing: summary article. A report of the American College of Cardiology/American Heart Association Task Force on Practice Guidelines (Committee to Update the 1997 Exercise Testing Guidelines). J Am Coll Cardiol. 2002:40(8):1531-40.

32. American College of Sports Medicine. ACSM's Guidelines for Exercise Testing and Prescription. 7th ed. Philadelphia: Lippincott Williams \& Wilkins; 2006.

33. Jones NL. Clinical Exercise Testing. 3rd ed. Philadelphia: W.B Saunders; 1988.

34. Widrick JJ, Freedson PS, Hamill J. Effect of internal work on the calculation of optimal pedaling rates. Med Sci Sports Exerc. 1992;24(3):376-82.

35. Zoladz JA, Rademaker AC, Sargeant AJ. Human muscle power generating capability during cycling at different pedalling rates. Exp Physiol. 2000;85(1): $117-24$.

\section{Submit your next manuscript to BioMed Central and we will help you at every step:}

- We accept pre-submission inquiries

- Our selector tool helps you to find the most relevant journal

- We provide round the clock customer support

- Convenient online submission

- Thorough peer review

- Inclusion in PubMed and all major indexing services

- Maximum visibility for your research

Submit your manuscript at www.biomedcentral.com/submit

CBiomed Central 